\title{
Reversible photobleaching of photoconvertible SAASoti-FP
}

\author{
Ilya D. Solovyev ${ }^{1,2}$, Alexandra V. Gavshina², Alexander P. Savitsky ${ }^{1,2 *}$ \\ ${ }^{1}$ Department of Chemistry, M.V. Lomonosov Moscow State University, Leninskie Gory 1/3, Moscow 119991, \\ Russian Federation \\ ${ }^{2}$ A.N. Bach Institute of Biochemistry, Research Center of Biotechnology of the Russian Academy of Sciences, \\ Leninsky Ave. 33, bld. 2, Moscow 119071, Russian Federation
}

* e-mail: apsavitsky@inbi.ras.ru

\begin{abstract}
SAASoti is a green fluorescent protein suitable for use in super-resolution microscopy as it can be photoconverted to red fluorescence under $405 \mathrm{~nm}$ illumination. The green fluorescence of V127T SAASoti variant is reversibly photobleached under exposure to $470 \mathrm{~nm}$ light without photoconversion to the red form. The phenomenon can be explained by chromophore protonation that was confirmed by an increase in absorption at $400 \mathrm{~nm}$ (chromophore protonated form) and a decrease at $509 \mathrm{~nm}$ (anionic form). This light-induced photoswitching can be repeated with the same sample several times without loss of the initial fluorescence intensity. Subsequent sample exposures result in the same fluorescence recovery process. By changing the content of the $\mathrm{H}$-form one can control photoswitching as only the protonated SAASoti may be converted to the red form. This property is extremely important for sub-diffraction microscopy. (C) 2017 Journal of Biomedical Photonics \& Engineering.
\end{abstract}

Keywords: fluorescent proteins; photobleaching; reversible photoswitching.

Paper \#3260 received 16 Nov 2017; revised manuscript received 25 Dec 2017; accepted for publication 25 Dec 2017; published online 31 Dec 2017. doi: 10.18287/JBPE17.03.040303. [Saratov Fall Meeting 2017 Special Issue].

\section{References}

1. D. Shcherbakova, P. Sengupta, J. Lippincott-Schwartz, and V. V. Verkhusha, "Photocontrollable fluorescent proteins for superresolution imaging," Annual Review Biophysics 43, 303-329 (2014).

2. G. Lapshin, A. Salih, P. Kolosov, M. Golovkina, Y. Zavorotnyi, T. Ivashina, L. Vinokurov, V. Bagratashvili, and A. Savitsky, "Fluorescence color diversity of great barrier reef corals," Journal of Innovative Optical Health Sciences 8(4), 1550028 (2015).

3. S. Habuchi, P. Dedecker, J. Hotta, C. Flors, R. Ando, H. Mizuno, A. Miyawaki, and J. Hofkens, "Photoinduced protonation/ deprotonation in the GFP-like fluorescent protein Dronpa: mechanism responsible for the reversible photoswitching," Photochemical \& Photobiological Sciences 5(6), 567-576 (2006).

4. V. Adam, M. Lelimousin, S. Boehme, G. Desfonds, K. Nienhaus, M. J. Field, J. Wiedenmann, S. McSweeney, G. U. Nienhaus, and D. Bourgeois, "Structural characterization of IrisFP, an optical highlighter undergoing multiple photo-induced transformations," Proceedings of the National Academy of Sciences 105(47), 1834318348 (2008).

5. T. Brakemann, A. C. Stiel, G. Weber, M. Andresen, I. Testa, T. Grotjohann, M. Leutenegger, U. Plessmann, H. Urlaub, C. Eggeling, M. C. Wahl, S. W. Hell, and S. Jakobs, "A reversibly photoswitchable GFP-like protein with fluorescence excitation decoupled from switching," Nature Biotechnology 29, 942-947 (2011).

6. D. Bourgeois, "Deciphering structural photophysics of fluorescent proteins by kinetic crystallography," International Journal of Molecular Sciences 18(6), 1187 (2017).

7. J. Petersen, P. G. Wilmann, T. Beddoe, A. J. Oakley, R. J. Devenish, M. Prescott, and J. Rossjohn, "The 2.0- $\AA$ crystal structure of eqFP611, a far red fluorescent protein from the sea anemone Entacmaea quadricolor," Journal of Biological Chemistry 278(45), 44626-44631 (2003).

8. M. Andresen, M. C. Wahl, A. C. Stiel, F. Grater, L. V. Schafer, S. Trowitzsch, G. Weber, C. Eggeling, H. Grubmuller, S. W. Hell, and S. Jakobs, "Structure and mechanism of the reversible photoswitch of a fluorescent protein," Proceedings of the National Academy of Sciences 102(37), 13070-13074 (2005). 
9. M. Quillin, D. M. Anstrom, X. Shu, S. O'Leary, K. Kallio, D. M. Chudakov, and S. J. Remington, "Kindling fluorescent protein from Anemonia sulcata: dark-state structure at $1.38 \AA$ resolution," Biochemistry 44(15), 5774-5787 (2005).

10. H. Mizuno, P. Dedecker, R. Ando, T. Fukano, J. Hofkens, and A. Miyawaki, "Higher resolution in localization microscopy by slower switching of a photochromic protein," Photochemical \& Photobiological Sciences 9(2), 239-248 (2010).

11. T. Roebroek, S. Duwé, W. Vandenberg, and P. Dedecker, "Reduced fluorescent protein switching fatigue by binding-induced emissive state stabilization," International Journal of Molecular Sciences 18(9), 2015 (2017).

12. A. Rusanov, V. A. Mironov, A. S. Goryashenko, B. L. Grigorenko, A. V. Nemukhin, and A. P. Savitsky, "Conformational partitioning in $\mathrm{pH}$-induced fluorescence of the kindling fluorescent protein (KFP)," The Journal of Physical Chemistry B, 115(29), 9195-9201 (2011).

13. S. Gayda, K. Nienhaus, and G. Nienhaus, "Mechanistic insights into reversible photoactivation in proteins of the GFP family," Biophysical Journal 103(12), 2521-2531 (2012).

14. D. K. Tiwari, Y. Arai, M. Yamanaka, T. Matsuda, M. Agetsuma, M. Nakano, K. Fujita, and T. Nagai "A fastand positively photoswitchable fluorescent protein for ultralow-laser-power RESOLFT nanoscopy," Nature Methods. 12(6), 515-518 (2015).

15. H. Ai, and R. Campbell, "Teal fluorescent proteins: characterization of a reversibly photoswitchable variant," Proceedings of SPIE 6868, 68680D (2008).

16. X. He, A. Bell, and P. Tonge, "Ground state isomerization of a model green fluorescent protein chromophore," FEBS Letters 5491(1-3), 35-38 (2003).

17. V. Mironov, M. G. Khrenova, B. L. Grigorenko, A. P. Savitsky, and A. V. Nemukhin, "Thermal isomerization of the chromoprotein asFP595 and its kindling mutant A143G: QM/MM molecular dynamics simulations," The Journal of Physical Chemistry B 117 (43), 13507-13514 (2013).

18. A. Stiel, S. Trowitzsch, G. Weber, M. Andresen, C. Eggeling, S.W. Hell, S. Jakobs, and M.C. Wahl, “1.8 A bright-state structure of the reversibly switchable fluorescent protein Dronpa guides the generation of fast switching variants," Biochemical Journal 402(1), 35-42 (2007).

19. V. Adam, B. Moeyaert, C. C. David, H. Mizuno, M. Lelimousin, P. Dedecker, R. Ando, A. Miyawaki, J. Michiels, Y. Engelborghs, J. Hofkens "Rational design of photoconvertible and biphotochromic fluorescent proteins for advanced microscopy applications," Chemistry \& Biology 18(10), 1241-1251 (2011).

\section{Introduction}

Fluorescent proteins (FPs) have grown into indispensable tools for life science studies. Among them reversibly photoswitchable fluorescent proteins (RPFPs) are of special interest as they can be applied to superresolution fluorescence microscopy techniques [1].

Originally discovered in the Stylocoeniella armata coral, SAASoti FP was found to be irreversibly photoconvertible from green to red fluorescence under $405 \mathrm{~nm}$ illumination [2]. The process is accompanied by peptide bond break. Previously we have demonstrated the $\mathrm{pH}$-dependence of this photoconversion and the presence of protonated and anionic chromophore forms [2]. Wild type SAASoti exists as a tetramer, while V127T point mutation led to its monomerization.

On the one hand, $\beta$-barrel structure of fluorescent proteins protects the chromophore from different types of fluorescence quenching, but on the other hand, neighboring amino acid residues facing inside the barrel interact with the chromophore and can therefore affect its photophysical properties. The effect of photoinduced bleaching is known for different GFP -like proteins [3], [4]. In some cases it is associated with cis-trans isomerization of the chromophore and a change of its protonation state, while in the case of Dreiklang FP the chromophore undergoes reversible hydration by a completely different mechanism [5]. For another greento-red photoconvertible FP well-described today -
IrisFP - the photobleaching was shown to be reversible [4]. After X-ray structure analyses of the fluorescent ('on') and photobleached ('off') crystals the authors also determined amino acid residues that are crucial for stabilizing different states of the chromophore. Firstly, it was shown that the chromophore undergoes cis-to-trans isomerization during photobleaching. While the cisconfiguration is stabilized by the E144-H194-E212 Hbonded triad, it is replaced by the E144-R66-E212 triad in the case of the trans-configuration [4]. Accordingly, either H194 or R66 stabilizes the chromophore by $\pi$ stacking or $\pi$-cation interactions, respectively [6]. As there exist FPs with trans- chromophore in their 'on' state [7] it should be emphasized that it is not the configuration (cis- or trans-) but its protonation state that is responsible for the fluorescence. Here we report the phenomenon of reversible photoswitching observed for V127T SAASoti-FP.

\section{Materials and methods}

\subsection{Protein expression and isolation}

The recombinant plasmid pET22b containing V127TSAASoti gene (669 bp) was transformed into $E$. coli BL21 (DE3) cells, cultured at $37{ }^{\circ} \mathrm{C}$ and $200 \mathrm{rpm}$ shaking in LB medium with $100 \mathrm{mkg} / \mathrm{ml}$ ampicillin to an optical density of 0.5 . Protein synthesis was induced 

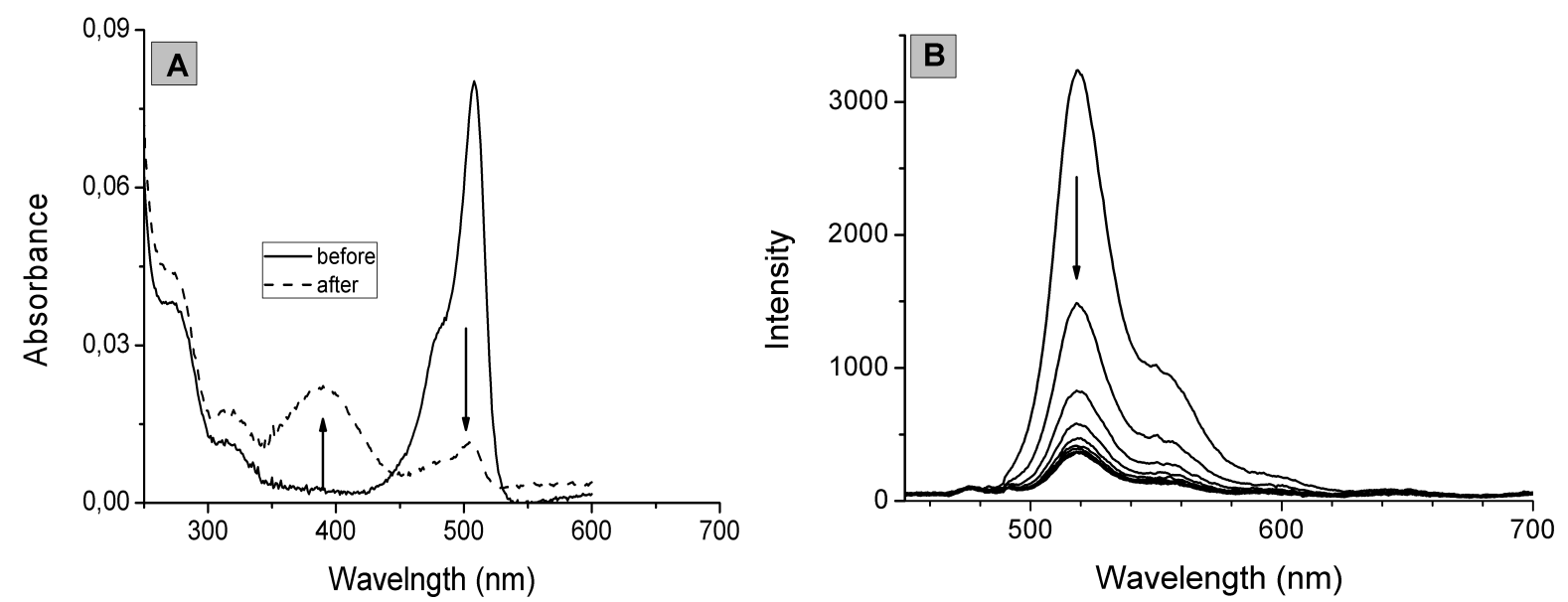

Fig. 1 (A) Absorbance spectrum of V127T SAASoti-FP variant before (solid line) and after (dashed line) $10 \mathrm{~min} 470$ $\mathrm{nm}$ exposure $\left(1 \mathrm{~W} / \mathrm{cm}^{2}\right)$; (B) Fluorescence spectra under $470 \mathrm{~nm}\left(1 \mathrm{~W} / \mathrm{cm}^{2}\right)$ exposure every minute during $10 \mathrm{~min}$.

by addition of IPTG to the final concentration of 0.05 $\mathrm{mM}$ and subsequent incubation for $24 \mathrm{~h}$ at $20^{\circ} \mathrm{C}$ and $200 \mathrm{rpm}$ shaking. After E. coli disruption with a French press, the bacterial cell pellet was suspended in $20 \mathrm{mM}$ Tris-HCL ( $\mathrm{pH} 7.5$ ) containing $150 \mathrm{mM} \mathrm{NaCl}, 1 \mathrm{mM}$ PMSF, and $1 \mathrm{mkg} / \mathrm{ml}$ DNAse. After ammonium sulfate was added to $50 \%$ saturation $(\mathrm{v} / \mathrm{v})$, the colored fraction was separated by centrifugation at $18000 \mathrm{~g}$ and $4{ }^{\circ} \mathrm{C}$. The colored precipitate was dissolved in $20 \mathrm{mM}$ $\mathrm{NaHCO}_{3}$. After overnight dialysis against $20 \mathrm{mM}$ $\mathrm{NaHCO}_{3}$ solution, the V127T SAASoti solution was further purified with MonoQ-based ion-exchange chromatography. The protein was eluted with a linear 0 $-0.5 \mathrm{M} \mathrm{NaCl}$ gradient performed by mixing $20 \mathrm{mM}$ $\mathrm{NaHCO}_{3}$ anion-exchange buffer (A) and $20 \mathrm{mM}$ $\mathrm{NaHCO}_{3}$ containing $0.5 \mathrm{M} \mathrm{NaCl}$ anion-exchange buffer (B). AKTA purifier chromatographic system (GE Healthcare) with multiwavelength detection $(260 \mathrm{~nm}$, $280 \mathrm{~nm}$ and $509 \mathrm{~nm}$ ) was used. Eluted fractions were analyzed by SDS-PAGE and concentrated.

\subsection{Spectroscopic experiments}

Prior to the spectroscopic experiments SAASoti was diluted in $200 \mathrm{mM}$ sodium phosphate buffer $\mathrm{pH} 8.0$ to the final concentration of $3 \mu \mathrm{M}$. The photobleaching and relaxation experiments were carried out in $3 \mathrm{~mm}$ Hellma quartz cuvettes. Illumination of the samples at $470 / 24 \mathrm{~nm}\left(1 \mathrm{~W} / \mathrm{cm}^{2}\right)$ was performed by LED Lumencor Spectra X. Fluorescence kinetics data were obtained with a homebuilt linear CCD spectrometer based on Sony ILX511. Light power was verified with a Newport 2936-c power meter. Absorption spectra for thermal relaxation were recorded on a Cary 300 Bio spectrophotometer (Varian). Data analysis was performed with Origin 8.5 software package.

\section{Results and discussion}

After exposing the V127T SAASoti variant to $470 \mathrm{~nm}$ light $\left(1 \mathrm{~W} / \mathrm{cm}^{2}\right)$ for $10 \mathrm{~min}$, we observed the phenomenon of photobleaching. As it can be noticed from the absorption spectra in Fig. 1A, $470 \mathrm{~nm}$ exposure during 10 min leads to a $395 \mathrm{~nm}$ absorbance peak appearance while the absorbance intensity of the green form $(509 \mathrm{~nm})$ decreases. Previously it has been shown that the protonated chromophore absorbs at 395 $\mathrm{nm}$ while its anionic form shows a maximum absorbance at $509 \mathrm{~nm}$. In other words, here we observe photoinduced switching between two different protonation states of the chromophore in the same buffer system ( $\mathrm{pH} 8)$.

It is known for different FPs that during photobleaching cis-to-trans isomerization of the chromophore takes place along with its protonation [4], [8], [9]. The process can be reversed either by illumination at $405 \mathrm{~nm}$ or during thermal relaxation. We investigated thermal relaxation as V127T SAASoti can be irreversibly converted into the red fluorescent form by $405 \mathrm{~nm}$ illumination. Fig. 2A shows that photobleaching followed by thermal relaxation of the probe can be repeated with the same sample several times without any significant changes in the fluorescence intensity of the green form at $520 \mathrm{~nm}$.

The photobleaching experiments with different fluorescent proteins exhibit bi- and mono-exponential fluorescence decay dependences [10], [11]. To characterize the photoswitching between 'on' and 'off' forms we recorded the photobleaching (Fig. 2A) and thermal relaxation (Fig. 3A) kinetics. A bi-exponential function (Eq. 1) fit the photobleaching experimental data well with $\mathrm{R}^{2}=0.999$ and the random distribution of the residuals (Fig. 2A).

$$
I=I_{1} * \exp \left(-k_{1} t\right)+I_{2} * \exp \left(-k_{2} t\right)+c
$$

where $I_{1}, I_{2}$ - pre-exponential coefficients, $c-$ background signal including residual SAASoti fluorescence. The calculated rate coefficients are $k_{1}=0.028 \mathrm{~s}^{-1}$ and $k_{2}=0.011 \mathrm{~s}^{-1}$ at $22{ }^{\circ} \mathrm{C}$ with the $1: 1$ ratio of the pre-exponential coefficients. The rate of 

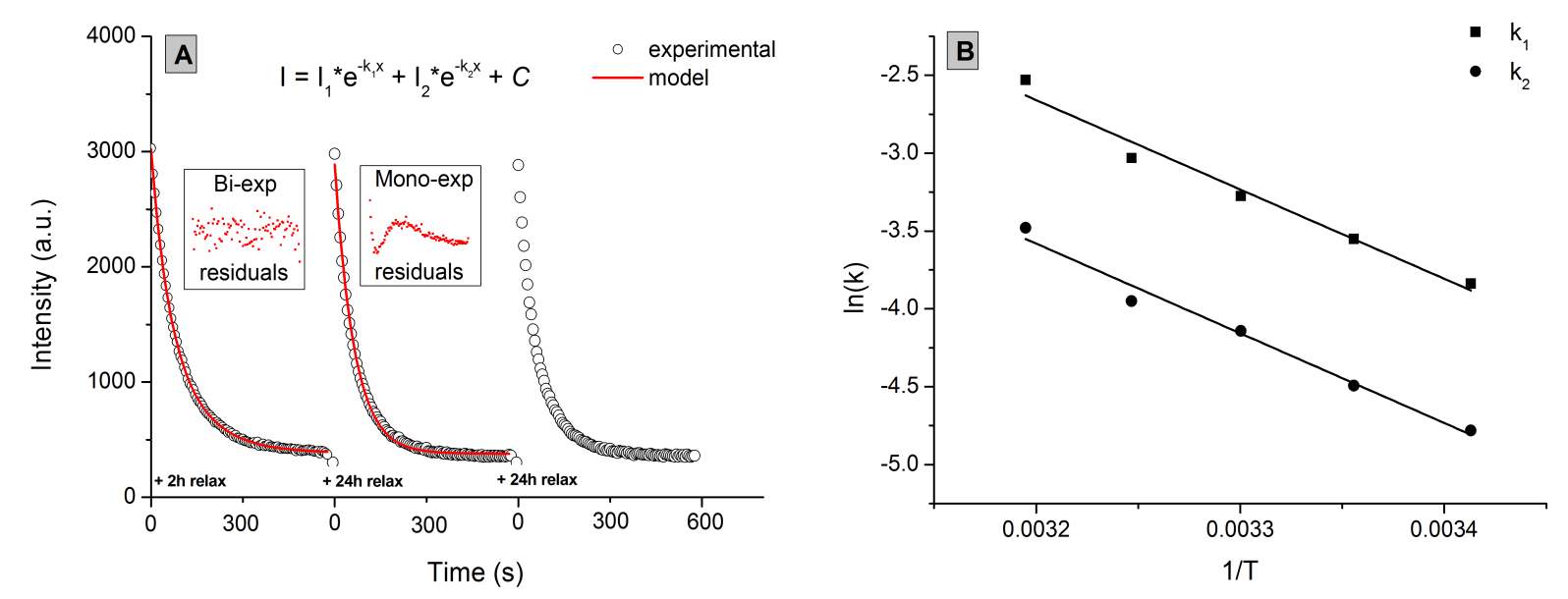

Fig. 2 (A) Photobleaching kinetics of V127T SAASoti-FP. Subsequent photobleaching cycles of the same sample including $470 \mathrm{~nm}$ exposure during $10 \mathrm{~min}\left(1 \mathrm{~W} / \mathrm{cm}^{2}\right)$ and $\sim 24 \mathrm{~h}$ thermal relaxation at $22^{\circ} \mathrm{C}$. Fitting of the data to a biexponential model. (B) Arrhenius plots of rate coefficients for the photobleaching kinetics.
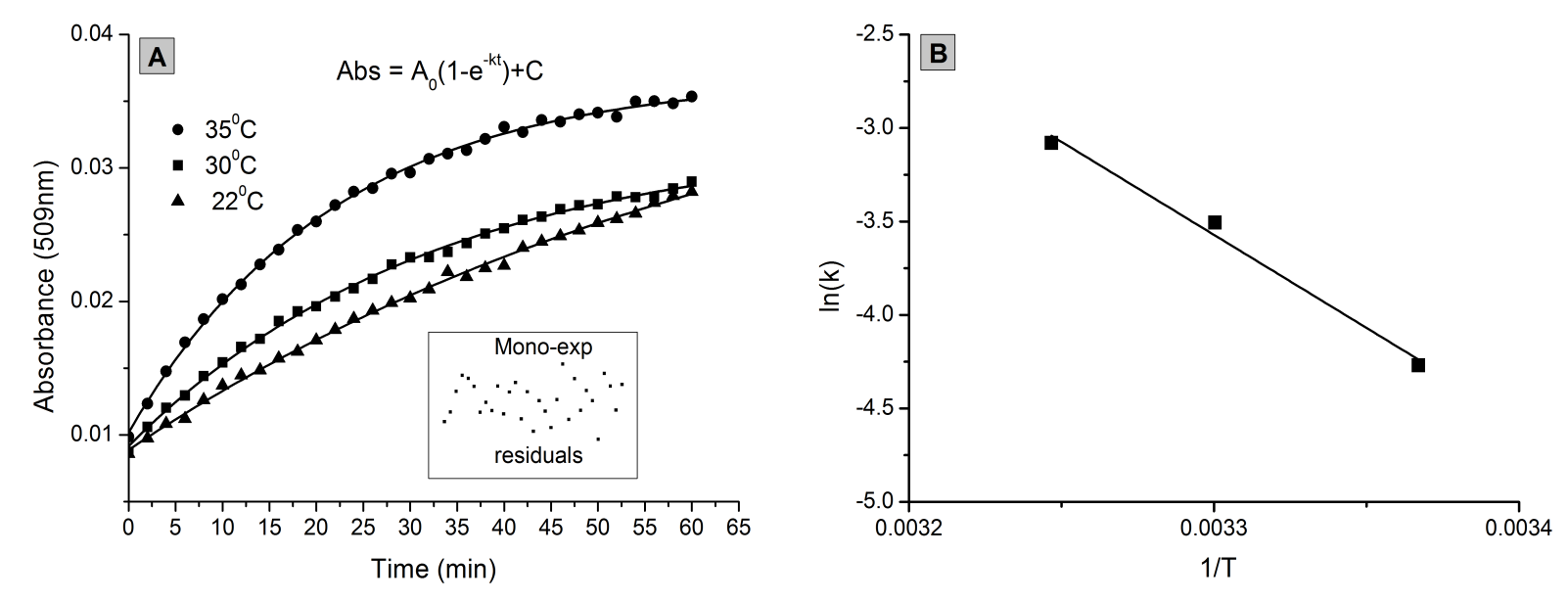

Fig. 3 (A) Thermal relaxation kinetics. The green form absorption recovery in time at different temperatures and fitting of the data to a mono-exponential dependency; (B) Arrhenius plot.

photobleaching certainly depends on the light exposure intensity. The phenomenon of bi-exponential fluorescence decay was explained by the existence of two protein populations in the excited state that can be reversibly converted to the 'off' state [11]. Previously, this 'multi protein populations' hypothesis was suggested by our group [12] when conducting experiments on KFP and its $\mathrm{pH}$-dependency. Sometimes it is difficult to compare bleaching kinetics data for different FPs as the system parameters (illumination intensity, beam shape, geometry, and concentration of the sample) in different cases can vary dramatically. In order to calculate the photobleaching activation energy (Ea) we illuminated the sample with $470 \mathrm{~nm}$ light at different temperatures in the range of $20-50{ }^{\circ} \mathrm{C}$. $E a$ was calculated from the Arrhenius equation (Eq. 2) as $47 \mathrm{~kJ} / \mathrm{mol}$ (Fig. 2B) for both protein populations.

$$
k=A * \exp \left(-E_{a} / k_{B} T\right)
$$

Fig. $3 \mathrm{~A}$ shows the relaxation kinetics recorded as a time dependency of the absorption recovery at $509 \mathrm{~nm}$ for a photobleached sample. Experiments on thermal relaxation reveal the mono-exponential dependence $\left(\mathrm{R}^{2}=0.998\right.$ and the random distribution of the residuals) of the process (Eq. 3)

$$
A=A_{0} *(1-\exp (-k t))+c
$$

with $k=0.014 \mathrm{~min}^{-1}$ at $22{ }^{\circ} \mathrm{C}$. The mono-exponential mechanism was also demonstrated in several works on FPs' thermal relaxation [13], [14]. Estimated $E a$ value for thermal relaxation ( $83 \mathrm{~kJ} / \mathrm{mol}$ ) (Fig. 3B) is obviously lower than that in the case of mTFP0.7 $(106 \mathrm{~kJ} / \mathrm{mol})$ [15] and Dronpa (109 kJ/mol) [3] but slightly higher than $55 \mathrm{~kJ} / \mathrm{mol}$ for a synthesized GFP-like chromophore [16]. KFP protein alternatively has a stable trans 'off' form and can thermally relax from the fluorescent cis 'on' form. Quantum mechanics/molecular mechanics 


\begin{tabular}{|c|c|c|c|}
\hline SAASoti & 1 & 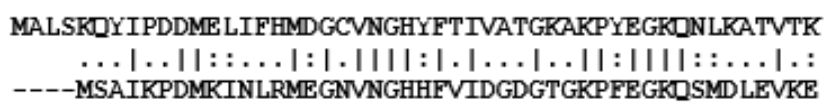 & 50 \\
\hline SAASoti & 51 & GAPLPFSTDILSTVMHYGNRCIVHYPPGI LDYFKDSFPEGYSWERTFAFE & 100 \\
\hline IrisFP & 47 & 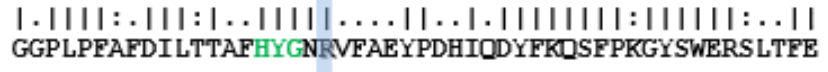 & 96 \\
\hline SAASoti & 101 & 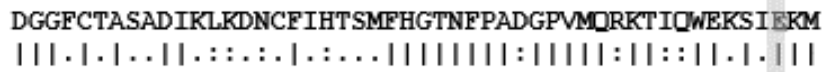 & 150 \\
\hline IrisFP & 97 & DGGICIARNDITMEGD TFYNKVRFHGVNFPANGPVMOKKT LKWEPST EKM & 146 \\
\hline SAASoti & 151 & 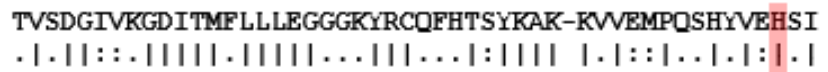 & 199 \\
\hline IrisFP & 147 & YVRDGVLTGDITMALLLEGNAHYRCDSRTT'YKAKEKGVKLPGYHLVDHCI & 196 \\
\hline SAASoti & & $\begin{array}{l}\text { E--RTNDDGTOFE LNEHAVARLNE------- } \\
\text { | } \ldots:|\ldots: .|,|||||| \ldots .\end{array}$ & \\
\hline IrisFP & 197 & EILSHDKDYNKVKLYFHAVAHSGLPDNARR & \\
\hline
\end{tabular}

Fig. 4 Amino acid sequence alignment of V127T SAASoti with IrisFP. Chromophore forming residues are shown in green, E144* and E212 capturing the chromophore in the cis-and trans-configuration at the same time are grey shaded, whereas R66 (trans-) and H194 (cis-) are shaded blue-grey and red, respectively. (Program: needle, Matrix: EBLOSUM62). * - IrisFP amino acid numbering.

(QM/MM) simulations of KFP cis-trans isomerization predicts $E a=86 \mathrm{~kJ} / \mathrm{mol}(20.7 \mathrm{kcal} / \mathrm{mol})$ [17] that is in good agreement with the previously published experimental data $(71 \mathrm{~kJ} / \mathrm{mol})[9]$.

Thermal relaxation of V127T SAASoti occurs with a $50 \mathrm{~min}$ half-life $\left(\tau_{1 / 2}\right)$ of the dark state at $22{ }^{\circ} \mathrm{C}$. In the case of mTFP0.7 [15], the fluorescence intensity recovers in $30 \mathrm{~min}$, thermal relaxation in the case of IrisFP [4] and Dronpa [18] occurs much slower with the $\tau_{1 / 2}$ values equal to $5.5 \mathrm{~h}$ and $14 \mathrm{~h}$, respectively. Much faster relaxation kinetics in comparison with IrisFP and Dronpa along with the lower $E a$ values of SAASoti may indicate that V127T SAASoti is less stable in its 'off'state.

Amino acid sequence alignment of V127T SAASoti with well-studied IrisFP (Fig. 4) reveals over 64\% sequence similarity and the same positions of the conserved amino acid residues - R66, E144, H194, and E212 (IrisFP a.a. numbering). Therefore, we suggest that the same residues capture the chromophore in the cis- and trans-configurations in the case of SAASoti-FP.

The F173S point mutation of green-to-red photoconvertible EosFP leads to the appearance of the reversible photoswitching phenomenon (IrisFP) [4]. By performing rational design of the chromophore environment (I157, M159, F173), the authors [19] later revealed that the same point mutation (F173S) in the case of Dendra2 also lead to the on/off photoswitching (NijiFP). Another promising mutation - M159A - in the case of EosFP resulted in the appearance of the on/off photoswitching along with a loss of the ability to be converted into the red form. The M159A variant of Dendra2 becomes photoswitchable and is still photoconvertible. Interestingly, in V127T SAASoti-FP, unlike EosFP and Dendra2, methionine occupies position 159 and phenylalanine - position 173, and the protein demonstrates on/off photoswitching.

\section{Conclusions}

Previously V127T SAASoti was shown to be irreversibly converted from green to red fluorescent form under $405 \mathrm{~nm}$ illumination. In this study we have shown for the first time that V127T SAASoti - FP without any point mutations in the chromophore environment - can be reversibly photoswitched from the green 'on' to the dark 'off' state. Studies of different photoswitching FPs have identified several amino acid residues crucial for formation of the on/off states of the chromophore. Some of them (R66, H194, E144, E212) are located at the same positions in the V127T variant of SAASoti. Curiously, V127T SAASoti is already photoswitchable without F173S and M159A substitutions, which are responsible for the appearance of the photoswitching phenomenon in the case of IrisFP and NijiFP. Although the mechanism of the photoswitching of V127T SAASoti has not been investigated in the study and remains unclear, it is most likely caused by cis-trans isomerization of the chromophore associated with the change of its protonation state.

We suggest that a further study of the different SAASoti-FP variants, on the one hand, can contribute a lot to the development of the super-resolution techniques, but on the other hand, will deepen our understanding of the photobleaching mechanisms.

\section{Disclosures}

The authors declare that there are no conflicts of interest related to this article.

\section{Acknowledgements}

The work was supported by Russian Science Foundation (grant number: 15-14-30019). 